Pathologe 2010 - [Suppl 2] 31:305-306

DOI 10.1007/s00292-010-1346-6

Online publiziert: 2. September 2010

(c) Springer-Verlag 2010

\section{P. Möller}

Institut für Pathologie, Universität Ulm

\title{
Arbeitsbericht der AG Hämatopathologie
}

Die diesjährige Sitzung der Arbeitsgemeinschaft (AG) Hämatopathologie fand wie schon in den vergangenen Jahren viele Interessenten. Erfreulicherweise waren im Auditorium wieder auffallend viele jüngere Kolleginnen und Kollegen anwesend, wobei auch in dieser Sitzung mittlerweile Kolleginnen erfreulich zahlreich vertreten waren. Von den angemeldeten Beiträgen zur Thematik konnten bei gedrängtem Programm 18 Beiträge vorgetragen werden.

Traditionell begann das Programm mit neuen Forschungsergebnissen aus dem Formenkreis der myeloischen Dys- und Neoplasien:

Andrulis et al. (Heidelberg) stellten einen von der Arbeitsgruppe Zentgraf (Deutsches Krebsforschungszentrum/ DKFZ) generierten monoklonalen Antikörper vor, mit dem man die Isozitratdehydrogense-1-Mutation $\mathrm{R} 132 \mathrm{H}$ immunhistologisch darstellen kann. Damit gelingt es erstmals, mit einer einfachen Immunhistologie diese Mutation im Myelodysplasiesyndrom zu erkennen. In einem Fall wurde die Mutation auch durch Sequenzierung des entsprechenden Genlokus verifiziert (s. Andrulis et al., Leukemia Res., 2010).

Sotlar et al. (München) berichteten über das bislang unkommentierte Phänomen der aberranten $\mathrm{CD}_{3} \mathrm{O}$-Expression in den neoplastischen Zellen der Mastozytose. In einer großen Fallserie konnten sie aufzeigen, dass $\mathrm{CD}_{3}$ o präferenziell von den aggressiven Formen der Mastozytose exprimiert wird und insofern als Marker für Aggressivität der Erkrankung benutzt werden kann, wobei sie darauf hinwiesen, dass die Mastzellnatur in jedem Fall über Marker wie Mastzelltryptase oder CD117 abgesichert werden sollte. Als funktionelles Modell stellten sie die HMC1Mastzelllinie vor, die ebenfalls CD3o-positiv sei.

Hussein et al. (Hannover) diskutierten die Brauchbarkeit der Nachweismethoden der $c$-Kit-D818V-Mutation bei der Mastozytosediagnostik. Die Erfahrungen dieser Arbeitsgruppe ist, dass die cDNA-Analyse sensitiver als die DNA-Analyse ist, wobei man, um sicher zu gehen, beide Methoden parallel einsetzten sollte.

Siebold et al. (Leipzig) demonstrierten ihre Befunde, dass die allelspezifische PCR („Wt-blocking QPCR“) ein sehr gutes Instrument ist, um mittels Nachweis der Jak2-V617F-Mutation eine minimale Resterkrankung des myeloproliferativen Syndroms nach Stammzelltransplantation nachzuweisen oder auszuschließen. Sie schlagen monatliche Kontrollen in den ersten 6 Monaten nach Stammzelltransplantation vor. Dieser Beitrag ist im Wesentlichen publiziert (Siebold et al., J Clin Pathol, 2010).

May et al. (Freiburg) untersuchten das Knochenmark von 6 Patienten mit akuter myeolischer Leukämie (AML), die mit dem Plk1-Inhibitor BI2536 behandelt worden waren. Sie beobachteten als Therapieeffekte atypische Mitosen mit zytoplasmatisch falsch lokalisierten Plkı („mitotische Katastrophen") und viele Apoptosen und konnten vermehrt aktivierte Caspase 3 nachweisen: Befunde, die darauf hin deuten, dass die Plk1-Inhibition zum für die Leukämiezelle tödlichen Verlust der orthotopen Plk1-Funktion führt.
Kapp-Schwörer et al. (Freiburg, Basel, Berlin, Düsseldorf) berichteten über ihre Erfahrungen mit dem noch unpräzisen Krankheitsbild der akuten Panmyelose mit Myelofibrose (APMF). Sie zeigten, dass die APMF eine deutlich schlechtere Prognose hat als die refraktäre Anämie mit Blastenexzess und Fibrose (RAEBF). Die Jak2-V617F-Mutation fanden sie in $36 \%$ der APMF und in $21 \%$ der RAEBF; aberrantes NPM1 fanden sie in $12,9 \%$ der APMF und in $7 \%$ der RAEBF. Die lebhafte Diskussion dieses Beitrags zeigte, dass die Hämatopathologen mit beiden Diagnosen noch ihre Probleme haben.

Engelhardt et al. (Hannover, es sprach Herr Bock) stellten eine eindruckvolle „Laser-scanning-microscopy-Untersuchung “ der Plättchenformierung vor. Megakaryozyten und die sich aus Pseudopodien dieser Zellart entwickelnden Proplättchen waren im Kontext der primären Myelofibrose die einzige Quelle des profibrotischen Thrombospondin-1.

Hussein et al. (Hannover) stellten Unterschiede im microRNA-Spektrum der chronischen myelomonozytären Leukämie (CMML) und der atypischen chronischen myeloischen Leukämie (aCML) vor. Bemerkenswert war, dass miR-424 in aCML, miR-223 in CMML und miR155 in CML jeweils um etwa den Faktor 5 im Vergleich zur Normalsituation erhöht war, während miR-10a in allen 3 Entitäten leicht herabreguliert erschien.

Es folgte ein interessantes Intermezzo aus dem Gebiet der Thymompathologie:

Marx et al. (Mannheim) zeigten Genexpressionsprofile von Thymomen und von normalen isolierten kortikalen und 
medullären Thymusepithelien. Die hierarchische Cluster-Analyse reproduziert die WHO-Klassifikation der Thymome. Die meisten Thymome zeigen kortikale und medulläre Signaturen, die Typ-AThymome waren dabei eher Medulla-typisch, die B1- und B2-Thymome waren erstaunlicherweise eher medullär, die ABThymome eher Kortex-typisch in ihren Expressionsprofilen.

Weiter im Programm ging es mit Lymphknotenpathologie und Lymphomen:

Wörner et al. (Würzburg) präsentierten eine vergleichende Studie von 40 Fällen mit „atypische EBV-assoziierter Lymphoproliferation" (AEAL) mit 65 akuten infektiösen Mononukleosen (AIM). Als Merksatz für die Praxis gilt, dass AIM selten B-Zell-seitig klonal sind und eine günstige Prognose haben, AEAL sind häufiger T-klonal oder B-klonal und nehmen einen nicht vorhersehbaren Verlauf, von Spontanremission bis zum hochmalignen Lymphom reichend.

Gattenlöhner et al. (Würzburg) berichteten über Neuigkeiten aus dem angestammten wissenschaftlichen Arbeitsgebiet, dem Transkriptionsfaktor RUNX1 (AML1). Sie stellten bislang nicht bekannte Isoformen vor und konnten zeigen, dass die RUNX1-Isoformen p48/p30 mit den Transkriptionsfaktoren FoxP 3 und NFAT interagieren und darüber Interleukin-2 regulieren.

Joosten et al. (Berlin) präsentierten neue Ergebnisse aus den pangenomischen Demethylierungs- und Histonacetylierungs-Experimenten von Herrn Hummel. Als Ergebnis zeigte sich, dass die kombinierte Demethylierung und Histonacetylierung von malignen T-Zell-Linien eine ALCL-ähnliche Signatur hervorbringt mit weitgehendem Verlust der T-Zell-Identität. ALCL-Linien indessen revertierten dadurch nicht zu T-Neoplasien.

Barth et al. (Ulm) stellten für die Lymphknoten-Referenzpathologien Frankfurt, Kiel, Lübeck, Berlin, St. Gallen, Würzburg/Stuttgart und Ulm einen FISHRingversuch zum Nachweis wichtiger Translokationen in der Lymphomdiagnostik vor, die dieses Konsortium durchgeführt hatte. Das Ergebnis war, dass alle gut und reproduzierbar die getesteten Bruchpunkte erkennen - und das un- ter Einsatz unterschiedlicher Techniken und Sonden(-kombinationen). Es folgte auf diesen Beitrag eine fast heftig zu nennende Diskussion.

Keller et al. (Berlin; es sprach Herr Loddenkemper) informierten über den Immunphänotyp und den Mutationsstatus der chronischen lymphatischen Leukämie (CLL) im Lymphknoten und fokussierten auf die plasmozytoide Differenzierung, die in etwa 10\% der CLL vorliegt. Unerwarteterweise waren die meisten CLL mit plasmozytoider Differenzierung unmutiert. Die vorläufige Analyse der klinischen Daten scheint dafür zu sprechen, dass die plasmozytoide Differenzierung ein ungünstiges prognostisches Kriterium sein könnte.

Adam et al. (Tübingen) wiesen mit ihren Untersuchungen darauf hin, dass in $15 \%$ der diffusen, großzelligen Lymphome und in $18 \%$ der Richter-Transfomationen Cyclin D1 immunhistologisch nachweisbar ist, aber nicht, wie beim Mantelzelllymphom durchgehend stark, sondern in wechselnder Antigendichte in unterschiedlich großen Subpopulationen. Diese Positivität ist nicht Folge einer $t(11 ; 14)$ sondern muss auf einem anderen hochregulierenden Prinzip beruhen.

Anagnastopoulos et al. (Berlin) stellten ihre Ergebnisse der immunhistochemischen Untersuchung von Chemokinrezeptoren im mediastinalen B-Zell-Lymphom (PMBL) vor. Das PMBL hat seltsam wenig CXCR5 und CCR7 und kein CCR6, dafür aber häufig CCR9. Mit diesem Markerprofil lässt sich das PMBL von anderen diffusen, großzelligen B-NHL und dem klassischen Hodgkin-Lymphom unterscheiden. Es wurde spekuliert, dass die niedrige Expression von CCR7 und CX$\mathrm{CR}_{5}$ für das lokal bleibende Wachstum und die fehlende Lymphknotendissemination verantwortlich sein könnte.

Oschlies et al. (Kiel, Giessen) stellten die größte existierende immunhistologisch analysierte Kohorte pädiatrischer lymphoblatischer Lymphome (LB) einer prospektiven, randomisierten Studie vor. $\mathrm{PAX}_{5}$ erwies sich als sensitiver und spezifischer Marker für die B-Linien-Zugehörigkeit, CD1a für die T-Linien-Zugehörigkeit. TdT ist ein Marker, der zwischen dem kortikalen und medullären Reifungsstadium einer T-ALL/eines T-LB diskriminiert.
Die in der Klassifikation geforderte Unterscheidung zwischen zytoplasmatischer und membranassoziierter $\mathrm{CD}_{3}$-Positivität kann die Immunhistologie nicht verlässlich leisten.

Dimitrova et al. (Berlin, Kiel) transfizierten die Hodgkin-Zelllinie L428 mit einem $P A X$-5-haltigen Vektor. Diese ektop PAX-5 exprimierende Hodgkin-Zelle rekonstituierte ihren B-Zell-Phänotyp nicht. Eine zusätzliche Behandlung dieser Transfektante mit demethylierenden und histonacetylierenden Substanzen führt auch nicht dazu, sondern „nur“ zur Heraufregulation von „Cancer-Testis-Antigenen“. Dies bedeutet, dass PAX-5 allein die B-Zell-Identität nicht aufrechterhalten kann.

Die daran anschließende Mitgliederversammlung stellte sich der letztjährig geäußerten Aufforderung, über einen neuen Sprecher der AG zu befinden. Der Vorschlag von Frau Prof. Schmitt-Graef, dass der aktuelle Sprecher weiter im Amt bleiben solle, fand breite Unterstützung unter den AG-Mitgliedern. Dies führte dazu, dass dieser seine Bereitwilligkeit bekundete, die AG weiter zu organisieren und ggf. nach außen zu vertreten und sich bei den Versammelten für das ihm entgegengebrachte Vertrauen bedankte. Dem drohenden Mitgliederschwund z. B. durch Emeritierung wichtiger Fachvertreter entgegenzuwirken, galt eine unangekündigte „Ex-cathedra-Entscheidung“ des Sprechers, alle anwesenden Akademikerinnen und Akademiker zu Mitgliedern zu küren. Diese Maßnahme rief spontan gute Laune und große Zustimmung unter den Anwesenden hervor. Die AG Hämatopathologie ging somit deutlich gestärkt aus der diesjährigen Sitzung heraus.

\section{Korrespondenzadresse \\ Prof. Dr. P. Möller}

Institut für Pathologie, Universität Ulm Albert-Einstein-Allee 11, $89081 \mathrm{Ulm}$ peter.moeller@uniklinik-ulm.de

Interessenkonflikt. Der korrespondierende Autor gibt an, dass kein Interessenkonflikt besteht. 